\title{
Association between macrophage activation and function of micro-encapsulated rat islets
}

\author{
P. de Vos, I. Smedema, H. van Goor, H. Moes, J. van Zanten, S. Netters, L. F. M. de Leij, A. de Haan, \\ B. J. de Haan \\ Transplantation Biology, Department of Pathology and Laboratory Medicine, Section of Medical Biology, \\ University of Groningen, Groningen, The Netherlands
}

\begin{abstract}
Aims/hypothesis. Survival of microencapsulated islet grafts is limited, even when inflammatory reactions against the capsules are restricted to a small portion of less than $10 \%$.

Methods. This study investigates both in vivo in rat recipients and in vitro whether cellular overgrowth on this minority of the capsules contributes to limitations in the functional survival of the $90 \%$ of the encapsulated islets which remain free of any cellular overgrowth. Results. In successful rat recipients of an allogenic microencapsulated islet graft we found that the vast majority of cells in the capsular overgrowth were activated ED-1 and ED-2 positive macrophages which were found in numbers of approximately 1500 per capsule. Co-culture of encapsulated islets with 1500 (nr8383) rat-macrophages per capsule showed that the activa-
\end{abstract}

tion of macrophages was caused by islet-derived bioactive factors since TNF- $\alpha$ and IL- $1 \beta$ secretion by macrophages was induced by islet-containing capsules and not by empty capsules. This activation of macrophages was associated with a decrease in function of the encapsulated islets as evidenced by a quantitatively reduced $(35 \%)$ insulin response in static incubation and a slower response in perifusion.

Conclusion/interpretation. Present research aims to design strategies for the temporary inhibition of macrophage activation since macrophages are predominantly present in the first two months after implantation. These strategies will serve as a pertinent basis for future clinical application of microencapsulated islets. [Diabetologia (2003) 46:666-673]

Keywords Islets, transplantation, alginate, poly-l-lysine, encapsulation, immunoisolation.
Immunoprotection involves envelopment of tissue in a semi-permeable membrane to protect donor cells against antibodies and cytotoxic cells of the host immune system. This immunoprotection by encapsulation allows for successful transplantation of cells in the absence of immunosuppression. A commonly used procedure for immunoprotection is microencapsula-

Received: 14 October 2002 / Revised: 13 December 2002

Published online: 15 May 2003

(C) Springer-Verlag 2003

Corresponding author: P. de Vos PhD, Transplantation Biology, Department of Pathology and Laboratory Medicine, Section of Medical Biology, University of Groningen, Hanzeplein 1, 9700 RB Groningen, The Netherlands

E-mail: p.de.vos@med.rug.nl tion of tissues in alginate-poly-l-lysine (PLL) based capsules [1]. During recent years, important advances have been made with this technology. The first allotransplantations in humans with encapsulated parathyroid cells and pancreatic islets have been successfully carried out $[2,3]$. Also, it has been shown to allow for successful xenotransplantation of islet grafts in both chemically induced and autoimmune diabetic rodents [4], dogs [5], and monkeys [6]. Although this illustrated the principle applicability of the alginate-encapsulation technique, a fundamental barrier has to be overcome since graft survival varies considerably from several days to months [4]. This variation in success rate is usually attributed to insufficient biocompatibility of the applied capsules with overgrowth of the capsules by macrophages and fibroblasts [7] and necrosis of the islets as a consequence [8]. 
Many groups, including ours, have focused in previous years on deletion of the tissue responses against encapsulated islets. This has resulted in many technical modifications to the procedure such as application of pure alginate [9], new types of alginate [10] and a better technology to completely envelop the islets [11, 12]. This progress has brought about a substantial reduction in the tissue responses against encapsulated grafts which has been reduced from a severe reaction affecting the majority of more than $90 \%$ of the encapsulated islets $[9,13]$ to a mild reaction which involves not more than a portion of 2 to $10 \%$ of the capsules [8, 13]. It is generally assumed that the loss of islets associated with this small portion of overgrown capsules has no consequences for the function of the remaining 90 to $98 \%$ of the graft. Unfortunately, the reduction in tissue reactions did not bring about the expected permanent survival of the microencapsulated islet grafts since survival of the grafts is still limited to periods of 4 to 9 months $[13,14,15]$.

Our study was undertaken to analyse the effect of overgrowth on 2 to $10 \%$ of the capsules on the function of islets in non-overgrown capsules. Therefore, we first quantified the number of macrophages in the capsular overgrowth in vivo, since this type of cell is predominantly found in the capsular overgrowth [13, 16]. These assessments were applied to design an in vitro system in which we compared the function of encapsulated islets with and without macrophages, by comparing insulin secretion during glucose challenge.

\section{Materials and methods}

Animals and induction of diabetes. Male inbred Albino Oxford $(\mathrm{AO} / \mathrm{G})$, Lewis rats, and Sprague-Dawley rats (SD) were obtained from Harlan (Harlan CPB, Zeist, The Netherlands) or the Central Animal Laboratory of Groningen. NHI-guidelines for the care and use of laboratory animals have been applied.

Male inbred Lewis-rats and SD-rats weighing 300 to 350 gram served as donors. Male inbred Albino Oxford rats (AO/G, the Central Animal Laboratory of Groningen, and The Netherlands) weighing 290 to 320 gram were used as recipients of encapsulated islet grafts. Diabetes was induced by injection of 75 to $90 \mathrm{mg} / \mathrm{kg}$ of streptozotocin (Zanosar, Upjohn, Kalamazoo, Mich., USA) via the tail vein as previously described [8]. To exclude beta-cell regeneration, we always took a biopsy from the pancreas at the time of peritoneal lavage [8].

Islet isolation and encapsulation. Islets were isolated according to a collagenase digestion method as previously described $[17,26]$. Islets were cultured in non-treated petri dishes (Greiner, Alphen a/d Rijn, The Netherlands) in portions of 100 islets per $25 \mathrm{~cm}^{2}$ for 19 to $44 \mathrm{~h}$ in RPMI-1640 [containing $10 \%$ foetal calf serum (FCS, Gibco, Breda, The Netherlands), $8.3 \mathrm{mmol} / \mathrm{l}$ glucose and $10 \mathrm{mmol} / \mathrm{l}$ Hepes and $1 \%$ Penicillium/Streptomycin] at $37^{\circ} \mathrm{C}$ in humidified air containing $5 \%$ $\mathrm{CO}_{2}$. The total islet volume was assessed by measuring the diameters [18] of islets in a $4 \%$ aliquot of the islet suspension. For transplantation experiments, total islet volume was at least $10 \mu \mathrm{l}$, which is approximately equal to the endocrine volume of the normal rat pancreas [19].
The alginate solution was converted into droplets using an air-driven droplet generator as previously described [20]. Polylysine-alginate encapsulation was carried out as described elsewhere [21]. Finally, the capsules had a diameter of 600 to $700 \mu \mathrm{m}$.

Transplantation and retrieval of microencapsulated islets. Under halothane anaesthesia, the microencapsulated islets were injected into the peritoneal cavity with a $16 \mathrm{G}$ cannula via a small incision $(3 \mathrm{~mm})$ in the linea alba. The abdomen was closed with a two-layer suture. The implanted volumes varied between 2.0 and $2.5 \mathrm{ml}$. Blood glucose was assessed at regular time intervals in blood sampled from the tail vein. The glucose concentration was assessed with glucose test tapes (Reflolux, Boeringer Mannheim, Germany). Recipients with a blood glucose level less than $8.4 \mathrm{mmol} / \mathrm{l}$ were considered normoglycaemic.

Microcapsules were retrieved at 4 weeks after transplantation as previously described [8]. Microcapsules were mostly freely floating and non-adherent, but occasionally we found some capsules adherent to the surface of intra-abdominal organs.

Histology and assessment of capsular overgrowth. Non-adherent capsules recovered by peritoneal lavage were always divided in two portions. We fixed one portion in pre-cooled $2 \%$ paraformaldehyde, buffered with $0.05 \mathrm{~mol} / \mathrm{l}$ phosphate, and processed for glycol methacrylate embedding [22]. Sections were prepared at $2 \mu \mathrm{m}$ and stained with Romanovsky-Giemsa stain. This portion was used to assess the degree of capsular overgrowth, which was quantified by expressing the number of recovered capsules with overgrowth as the percentage of the total number of recovered capsules for each individual animal. The second portion of the recovered capsules was frozen in pre-cooled iso-propaan, sectioned at $5 \mu \mathrm{m}$, and processed for immunohistochemical staining as previously described [12]. The monoclonal antibodies used were: a combination of ED1 and ED2 against monocytes and macrophages and subset of macrophages [23], HIS-40 against IgM-bearing B-lymphocytes [24], HIS-48 against neutrophilic granulocytes [25], R73 against $\mathrm{CD}^{+}$-bearing T-lymphocytes [26], ASIALO-GM1 directed to natural killer (NK) cells [27], and NOS6 against iNOS [28]. After several washing steps the sections were incubated with appropriate horseradish peroxidase-conjugated second step reagents. Peroxidase activity was shown by applying 3,3'-diaminobenzidine tetra HCL (DAB) containing hydrogenperoxide. In control sections, we used PBS instead of the first stage monoclonal antibody. This second portion was not only used to study the composition of the overgrowth but also to assess the number of ED-1 and ED-2 positive macrophages in the capsular overgrowth. To this end, we counted in each graft the macrophages on at least 100 sectioned overgrown capsules. Then we calculated for each graft the total number of cells on the overgrown capsules, considering the capsules to be perfect spheres. The cell number was expressed as a mean number of cells per recovered capsules for each individual animal.

Frozen sections were also applied to study iNOS-expression in macrophages obtained from in vitro experiments.

Processing for methacrylate embedding and RomanovskyGiemsa staining and for immunohistochemical staining as specified above was also applied to samples of adherent capsules recovered by excision.

Cell cultures and coincubation. Before starting the in vitro experiments we selected an appropriate macrophage-source for mimicking the in vivo situation. To this end, we first tested the feasibility of using residential macrophages either from over- 
grown capsules, from peripheral blood, or (proteose-peptone elicited) peritoneal macrophages [29]. This was done by comparing the number of iNOS-positive macrophages obtained from the in vitro-system with those collected in vivo on capsules directly fixed after being flushed from the peritoneal cavity. Unfortunately, none of the sources were adequate for application in the in vitro system since isolation and processing of the macrophages for in vitro application was associated with strong and inappropriate activation of the cells as evidenced by iNOS-expression in 80 to $95 \%$ of the macrophages (Fig. 1) while it was always less than $50 \%$ of the macrophages directly fixed after flushing from the peritoneal cavity. Another reason prohibiting the use of the above-mentioned macrophages as a cell source was that it was not applicable for long-term cultures since a substantial number of the macrophages died within hours after starting the incubations at $37^{\circ} \mathrm{C}$. Therefore, in our subsequent approach we applied the Sprague-Dawley-macrophage cell line NR8383 (ATCC, Manassas, Va., USA) [30] since processing for culture of this cellline was not associated with increased expression of iNOS and it was one of the few cell lines which was found to secrete IL-1 $\beta$ and TNF- $\alpha$ in a dose-dependent fashion upon activation with LPS.

In the co-incubation experiment, four separate samples of 100 encapsulated islets were incubated with an appropriate quantity of macrophages in 12-well plates and subsequently cultured for $48 \mathrm{~h}$ in RPMI-1640 with $10 \%$ foetal calf serum, $8.3 \mathrm{mmol} / \mathrm{l}$ glucose, $10 \mathrm{mmol} / \mathrm{l}$ Hepes, and $1 \%$ Penicillium/Streptomycin at $37^{\circ} \mathrm{C}$ in humidified air containing $5 \%$ $\mathrm{CO}_{2}$. As a negative control on macrophage activation we used cultures of macrophages without encapsulated islets. As positive controls served NR8383-macrophages activated with $1 \mu \mathrm{g} / \mathrm{ml}$ LPS. This amount of LPS maximally stimulates IL- $1 \beta$ and TNF- $\alpha$ production. At the end of the experiments, incubation media were removed and frozen for determination of IL-1 $\beta$ and TNF- $\alpha$. The cytokine secretory capacity by macrophages was expressed as $\mathrm{pg} / \mathrm{ml}$. Islets were harvested and processed for measurement of insulin secretion during glucose challenge.

Insulin secretion during glucose challenge. In static incubation experiments islets were tested in four separate samples of 10 islets each. To minimize the variability of the mean insulin responses, we selected islets with diameters between 150 and $200 \mu \mathrm{m}$. The encapsulated islets were preincubated for $45 \mathrm{~min}$ in $2 \mathrm{ml}$ Krebs-Ringer-bicarbonate (KRB), gassed with $95 \% \mathrm{O}_{2}$ and $5 \% \mathrm{CO}_{2}$, containing $0.25 \% \mathrm{BSA}$ and $2.75 \mathrm{mmol} / 1$ glucose. The quantitative insulin secretion was then assessed by three consecutive incubations: (i) $45 \mathrm{~min}$ in $2.75 \mathrm{mmol} / \mathrm{l}$ glucose in $\mathrm{KRB}$, (ii) $45 \mathrm{~min}$ in $16.5 \mathrm{mmol} / \mathrm{l}$ glucose in KRB (iii) $45 \mathrm{~min}$ in $2.75 \mathrm{mmol} / \mathrm{l}$ glucose in KRB. At the end of each incubation, the incubation media were removed and frozen for insulin determination. The insulin secretory responses were expressed as nanogram of insulin $\cdot \mathrm{ml}^{-1} \cdot 10$ islets $\mathrm{s}^{-1} .45 \mathrm{~min}^{-1}$.

In perifusion experiments islets were tested in three separate samples of 25 islets each. As with the static incubations, the variability of the mean insulin responses was minimized by selecting islets with diameters between 150 and $200 \mu \mathrm{m}$. Before the experiment the encapsulated islets were pre-perifused for $30 \mathrm{~min}$ in a modified cell perifusion apparatus at a flow rate of $0.9 \mathrm{ml} / \mathrm{min}$ with Krebs-Ringer-bicarbonate (KRB), gassed with $95 \% \mathrm{O}_{2}$ and $5 \% \mathrm{CO}_{2}$, containing $0.25 \% \mathrm{BSA}$ and $2.75 \mathrm{mmol} / \mathrm{l}$ glucose. The pattern or qualitative insulin secretion was then assessed by three separate perifusions: (i) $45 \mathrm{~min}$ in $2.75 \mathrm{mmol} / \mathrm{l}$ glucose in $\mathrm{KRB}$, (ii) $60 \mathrm{~min}$ in $16.5 \mathrm{mmol} / \mathrm{l}$ glucose + $0.05 \mathrm{mmol} / \mathrm{l} 3$-isobutyl-1-methylxanthine (IBMX) in $\mathrm{KRB}$, (iii) $45 \mathrm{~min}$ in $2.75 \mathrm{mmol} / \mathrm{l}$ glucose in $\mathrm{KRB}$. In the first and the second perifusion in low glucose, samples were col-

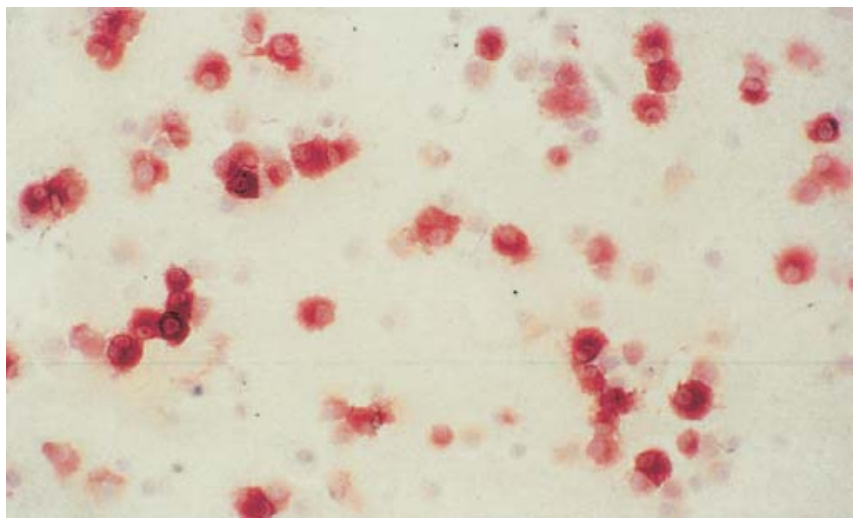

Fig. 1. Macrophages obtained from the peritoneal cavity. Note the positively stained macrophages expressing iNOS. (section of frozen material, stained for iNOS, original magnification $\times 300)$

lected every $5 \mathrm{~min}$. In the high glucose perifusion samples were collected at $0,1,2,3,4$, and 5 min after the start of the high glucose perifusion and every $5 \mathrm{~min}$ thereafter. Perifusion samples were removed and frozen for insulin determination. The insulin secretory responses were expressed as nanogram of insulin $\cdot \mathrm{ml}^{-1} .25$ islets $^{-1}$.

Determinations. Insulin was assessed by a radioimmunoassay for rat insulin (Linco, St. Charles, Mo., USA) using rat insulin as a standard. IL- $1 \beta$ and TNF- $\alpha$ were assessed by ELISA (R\&D, Abingdon, UK) using rat IL- $1 \beta$ and rat TNF- $\alpha$ as a standard.

Statistical analysis. Results are expressed as means \pm SEM. Statistical comparisons were made with the Mann Whitney U test. A $p$ value of less than 0.05 was considered statistically significant.

\section{Results}

Graft function and overgrowth of the capsule graft. All streptozotocin-induced diabetic AO-recipients of encapsulated Lewis-rat islets became normoglycaemic within 5 days after implantation. Transplantation of free, non-encapsulated Lewis-rat islets in streptozotocin-induced diabetic AO-recipients is always associated with immediate graft rejection. Encapsulated grafts were retrieved by peritoneal lavage at 4 weeks post implant.

At the time of peritoneal lavage, we occasionally found a portion of the capsules to be adherent to the omentum or liver. The vast majority of the capsules, however, was freely floating in the abdominal cavity without adhesion to the abdominal organs, as quantified by a retrieval rate of $91 \pm 5.3 \%$ of the capsules.

Virtually all capsules adherent to the abdominal organs were found to be overgrown by fibrotic tissue and occasionally by some macrophages. Of the $90 \%$ of capsules retrieved by peritoneal lavage, the range percentage of capsules with overgrowth varied between 2 to $10 \%$ with a mean of $8.1 \pm 0.5 \%$. The com- 
position of the overgrowth on free-floating capsules was different from that on capsules adherent to the abdominal organs. The free-floating capsules were surrounded by some fibrotic tissue but the vast majority

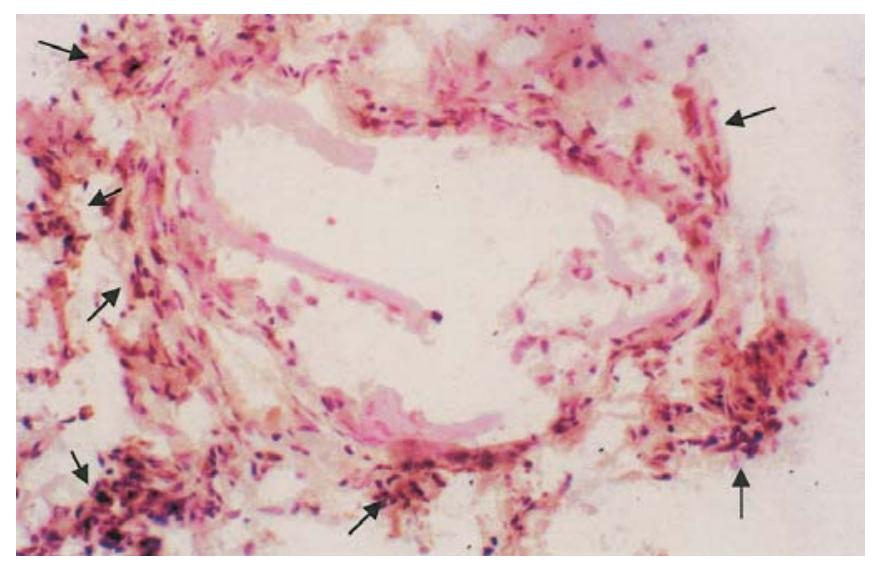

Fig. 2. Alginate-PLL capsules with capsular overgrowth, at one month post implant in rats. The arrows indicate macrophages. (section of frozen material, stained for the macrophage markers ED-1 and ED-2, original magnification $\times 200$ ) of cells were ED1- and ED2-positive macrophages (Fig. 2). We never observed NK-cells, granulocytes, CD3+-bearing T-lymphocytes, or B-lymphocytes cells.

In histological slices of free-floating capsules we quantified the number of ED-1 and ED-2 positive macrophages. The total number of macrophages varied between 11000 and 21000 per overgrown capsule. From these assessments follows that with an $8.1 \%$ overgrowth rate the quantity of macrophages per capsule amounts $1475 \pm 192$.

Since the islet in the capsule can, theoretically, influence the composition of the overgrowth, we evaluated whether the number of macrophages on empty capsules was different from that on isletcontaining capsules. With empty capsules the number of ED-1 and ED-2 positive macrophages was $1472 \pm 170$ per capsule which number is similar to the $1497 \pm 181$ found on islet-containing capsules. Therefore, in the next in vitro experiments we always applied 1500 macrophages per islet-containing capsule.
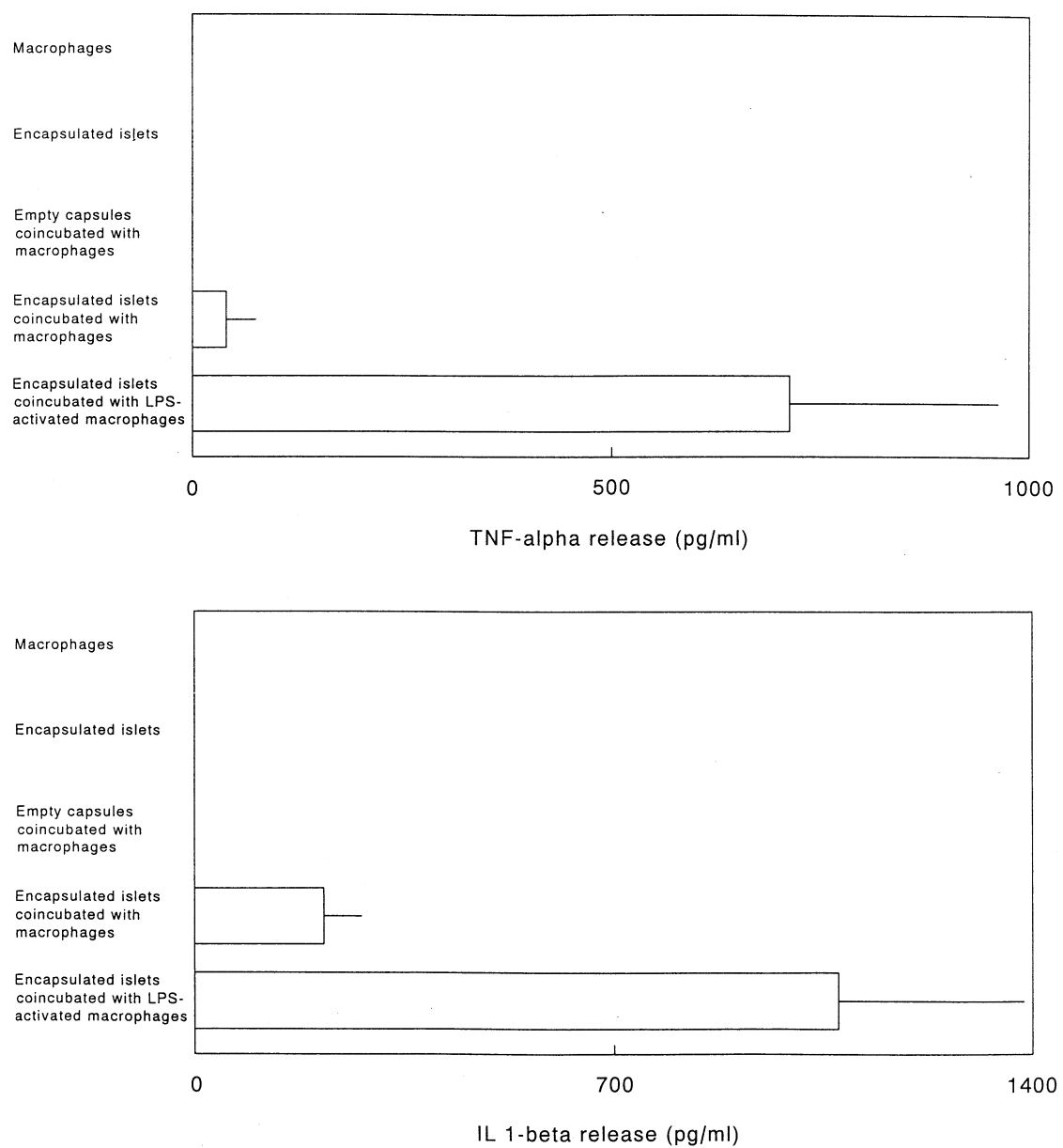

Fig. 3. IL-1 $\beta$ and TNF- $\alpha$ secretion by macrophages after $48 \mathrm{~h}$ of coincubation with various stimuli. The upper graph indicates the TNF- $\alpha$ production, the lower graph indicates the IL$1 \beta$ production. There was no secretion of cytokines by macrophages $(n=8)$ nor by islets $(n=6)$ when they were cultured alone in the absence of any stimuli. Empty capsules $(n=8)$ did not induce any response by macrophages. Encapsulated islets $(n=8)$ had an intermediate while LPS $(n=8)$ had a maximum effect on the cytokine production by macrophages. Values represent means \pm SEM 

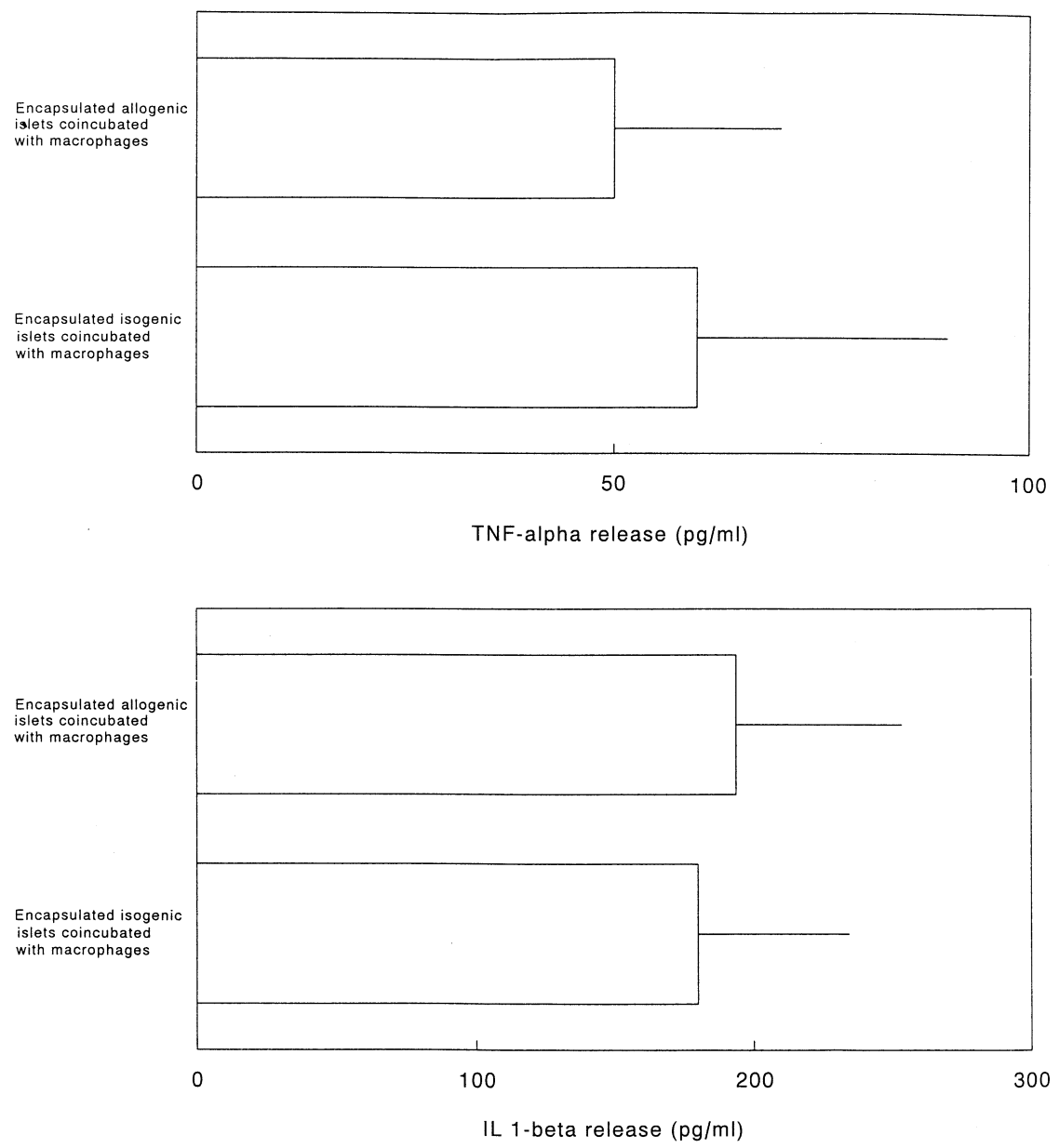

Fig. 4. IL-1 $\beta$ and TNF- $\alpha$ secretion by macrophages after $48 \mathrm{~h}$ of co-incubation with encapsulated allogenic islets $(n=6)$ and encapsulated isogenic islets $(n=3)$. Upper graph indicates the TNF- $\alpha$ production, the lower graph indicates the IL-1 $\beta$ production. Values represent means \pm SEM

Responses of rat macrophages on empty and isletcontaining capsules. Macrophage activation was quantified by measuring the production of the cytokines IL- $1 \beta$ and TNF- $\alpha$ after the administration of various stimuli. Media of cultures of macrophages alone and of islets alone, did not contain IL- $1 \beta$ or TNF- $\alpha$ (Fig. 3).

To investigate the effect of the capsules as such on the responses of macrophages, we coincubated empty capsules with macrophages. This experiment was repeated eight times but we never detected any of the two cytokines in the culture medium.

In order to assess the effect of an islet in capsules on the IL-1 $\beta$ and TNF- $\alpha$ production by macrophages, we compared the production of the cytokines in the following three cultures: cultures with encapsulated Lewis-rat islets alone, cultures of macrophages and encapsulated Lewis-rat islets, and cultures of LPSactivated macrophages and encapsulated Lewis-rat islets.
The allogenic islet in the capsules had a pronounced effect on the activation of macrophages. This effect was more prominent on the IL- $1 \beta$ production than on the TNF- $\alpha$ production since IL- $1 \beta$ production was always increased and was never absent while TNF- $\alpha$ was not found in the culture media of three out of eight co-incubation experiments. However, the effect of allogenic tissue on macrophage cytokine production was modest when compared to the effect of LPS on the IL-1 $\beta$-production $(214.8 \pm 63.5$ vs $1074.3 \pm 311.1 \mathrm{pg} / \mathrm{ml})$ and TNF- $\alpha$ production $(40 \pm 35$ vs $712.5 \pm 250 \mathrm{pg} / \mathrm{ml}$ ).

We questioned whether the macrophage responses were caused by the islets as such or by the histoincompatibility of the allogenic tissue. Therefore, we repeated the above experiments not only with Lewis ratislets, which imply an allogenic combination, but also with SD-islets, which implies an isogenic combination. The cytokine responses against allogenic islets were similar if not identical to the responses against isogenic islets (Fig. 4).

Insulin secretion by encapsulated islets after incubation with macrophages. The significance of the activation of macrophages on the function of the allogenic encapsulated islets was tested by carrying out glucose challenge experiments. This was done by static incubation to study the effects on the quantitative insulin 
production and by perifusion to assess the effect on the time-course of insulin secretion.

Co-incubation with macrophages had a clear-cut effect on the glucose induced insulin response of encapsulated islets (Fig. 5). When encapsulated islets were co-incubated with macrophages, we observed a reduction of approximately $35 \%$ of both the basal insulin secretion $(p<0.04)$ and the glucose-stimulated insulin secretion $(p<0.02)$. This effect of macrophages on insulin secretion was even more pronounced in co-incubations of encapsulated islets and LPS-activated macrophages, since islets in capsules had completely lost their ability to respond to a glucose load when LPS-activated macrophages were in their direct vicinity.

Since perifusion of encapsulated islets has been a subject of discussion, we decided to extend and to repeat the above experiments in order to compare the results of cultures of encapsulated islets not only with that of cultures with (LPS-activated) macrophages but also with that of cultures of free, non-encapsulated islets.

Free, non-encapsulated islets immediately responded with a rise in insulin secretion upon stimulation with glucose and showed a classical biphasic insulin response (Fig. 6). This response was different with encapsulated islets. Encapsulated islets responded slower but also with a lesser magnitude than the free, non-encapsulated islets. However, encapsulated islets did show a biphasic insulin response, although reduced and with a 12-min delayed first peak response when compared to free, non-encapsulated controls. It should be noted, however, that this increased insulin response of encapsulated islets at $15 \mathrm{~min}$ after starting the glucose load was repeatedly observed in the individual time curves but was not statistically different $(p<0.1)$.

The quantitative reduction of insulin secretion by co-incubation with macrophages is associated with a change in the pattern of insulin secretion. The islets cultured with macrophages immediately responded upon glucose and IBMX challenge with an increase in insulin secretion (Fig. 6). This response, however, was somewhat slower and reduced when compared to encapsulated islets cultured without macrophages. This slower response of encapsulated islets after incubation with macrophages is accompanied by an incomplete return to basal insulin secretion during the second incubation in low glucose. Encapsulated islets co-incubated with LPS-activated macrophages did not show any responsiveness to glucose and IBMX stimulation.

\section{Discussion}

Our report shows that the overgrowth of a minority and often neglected portion of not more than 2 to $10 \%$ of the capsules can have serious and detrimental ef-

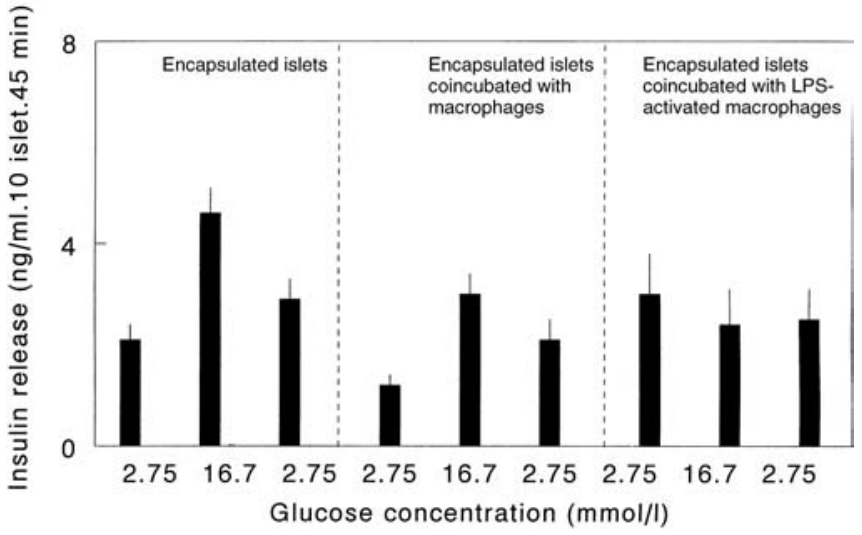

Fig. 5. Quantitative insulin response after glucose challenge (after static incubation) of encapsulated islets after $48 \mathrm{~h}$ of culture, of encapsulated islets $48 \mathrm{~h}$ co-incubated with macrophages, and of encapsulated islets $48 \mathrm{~h}$ co-incubated with LPSstimulated macrophages. The LPS maximally stimulates the cytokine production. All experiments were repeated six times. Values represent means \pm SEM

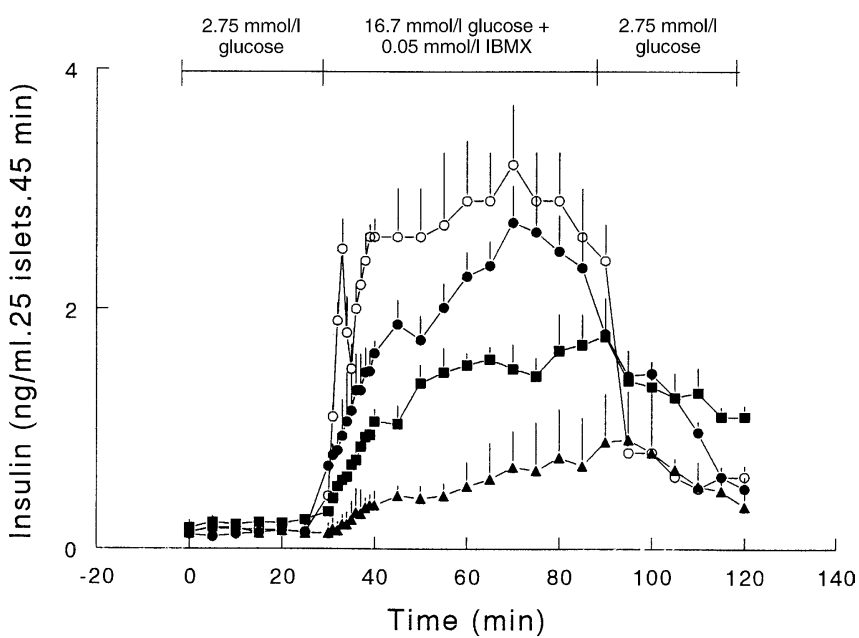

Fig. 6. Pattern of insulin secretion after glucose + IBMX challenge (after perifusion) of free, non-encapsulated controls and encapsulated islets after various co-incubations. The open circles $(n=6)$ are the insulin secretion by free, non-encapsulated islets. The closed circles $(n=5)$ are the insulin secretion by encapsulated islets after $48 \mathrm{~h}$ of culture alone, without macrophages. The closed boxes $(n=3)$ are the insulin secretion by encapsulated islets after $48 \mathrm{~h}$ of coincubation with macrophages. The closed triangles $(n=3)$ show the insulin secretion by encapsulated islets after $48 \mathrm{~h}$ of co-incubation with LPS-stimulated macrophages. The LPS maximally stimulates the cytokine production. Values represent means \pm SEM

fects on the function of encapsulated islets. This overgrowth in vivo on this small portion of capsules is caused by individual imperfections on the capsule surface [7] such as inadequate islet encapsulation [11] and incomplete covering of the inflammatory poly-1lysine [32]. Our study, also shows that the activation of attracted overgrowth is sustained by the islets in the capsules and not by the capsules themselves since cytokine production by macrophages in vitro is only 
increased when islets are inside the capsules and not when macrophages are cultured with empty capsules.

Our finding that encapsulated allogenic islets and encapsulated isogenic islets in capsules induce similar cytokine-responses in macrophages implies that factors other than alloantigens are responsible for the activation of macrophages. Possible candidates are cytokines produced by islet cells, which can readily pass the capsule membrane and induce inflammatory reactions [7]. It has been shown that islets without direct vascular access release inflammatory or angiogenic cytokines such as MCP-1 [33], and VEGF [34] which are known to stimulate macrophages. Another possibility is that components from necrotic islet cells leak out of the capsules and subsequently attract and activate macrophages. Several groups showing immune activation in the pancreas after gradual administration of beta-cell toxins support this suggestion [35].

Apparently, activated macrophages have a pronounced effect on the function of encapsulated islets. This explains our previous observations [8] and those of others [31], showing reduced insulin secretory capacity of encapsulated islet grafts with some overgrowth retrieved from the peritoneal cavity of recipients with a successful islet graft. Also, it corroborates the findings of another study [36], showing that transplantation of encapsulated islets in combination with local, intraperitoneal administration of agents selectively suppressing macrophages such as 15-deoxyspergualine is associated with a pronounced prolongation of the graft survival.

Our perifusion study shows that encapsulated islets respond upon a glucose challenge with a somewhat delayed and disturbed pattern when compared to free, non-encapsulated controls. These observations do not support the report of another study showing a biphasic insulin release by encapsulated islets which approach that of free, non-encapsulated islets [1]. This author, however, does not mention the size of the applied microcapsules, which is known to influence the rate and magnitude of the insulin response by encapsulated islets [37]. Our findings do confirm the results of one study [31] and the mathematical simulations of another $[38,39]$, showing that capsules of the size applied for transplantation are too large to allow for an undisturbed glucose-induced insulin response since the relatively low amount of insulin associated with the first-phase insulin release will largely dilute in the large volume of the capsule.

In the $90 \%$ of the capsules retrieved by peritoneal lavage only $60 \%$ of the initially grafted islets is found after 4 weeks of implantation [8]. This disappearance of islets is explained by the fact that the loss of islets associated with $10 \%$ of overgrown capsules retrieved by lavage is higher than this $10 \%$ since islet containing capsules are considered to have a higher change to be overgrown than empty capsules [8]. Our study provides an alternative explanation. As shown, grafting of encapsulated islets is associated with overgrowth of 2 to $10 \%$ of the capsules by activated and cytokine-releasing macrophages. This predictably interferes with survival of islets since the cytokines TNF- $\alpha$ and IL-1 $\beta$ have been shown to be potent stimulators of islet-cell death [40].

The fact that capsules can not adequately protect against cytokines such as IL-1 $\beta$ warrants some further consideration, since it should not be interpreted as an insurmountable obstacle for clinical application of microencapsulated islets. In a previous study, we have shown that the cellular overgrowth of the capsules is caused by defects on a small portion of capsules [13]. This cellular infiltrate was not a permanent but only a temporary phenomenon since after 2 months in the peritoneal cavity only overgrowth with fibroblasts surrounding the capsules was observed [13]. These observations imply that intervention is required in the first 2 months after implantation. Our efforts, therefore, focus on designing and testing immunosuppressive treatment strategies for temporary rather than permanently inhibiting macrophage activation. These strategies will serve as a pertinent basis for future clinical application of microencapsulated islets.

Acknowledgements. This work was supported by a grant from the J.F. de Cock Foundation and the Dutch foundation for scientific research.

\section{References}

1. Lim F, Sun AM (1980) Microencapsulated islets as bioartificial endocrine pancreas. Science 210:908-910

2. Soon Shiong P, Heintz RE, Merideth N et al. (1994) Insulin independence in a type 1 diabetic patient after encapsulated islet transplantation. Lancet 343:950-951

3. Hasse C, Klöck G, Schlosser A, Zimmermann U, Rothmund M (1997) Parathyroid allotransplantation without immunosuppression. Lancet 350:1296-1297

4. O'Shea GM, Sun AM (1986) Encapsulation of rat islets of langerhans prolongs xenograft survival in diabetic mice. Diabetes 35:943-946

5. Soon Shiong P, Feldman E, Nelson R et al. (1993) Longterm reversal of diabetes by the injection of immunoprotected islets. Proc Natl Acad Sci USA 90:5843-5847

6. Sun Y, Ma X, Zhou D, Vacek I, Sun AM (1996) Normalization of diabetes in spontaneously diabetic cynomologus monkeys by xenografts of microencapsulated porcine islets without immunosuppression. J Clin Invest 98:1417-1422

7. De Vos P, Van Schilfgaarde R (1999) Biocompatibility issues. In: Kühtreiber WM, Lanza RP, Chick WL (eds) Cell encapsulation technology and therapeutics. Birkhäuser, Boston, pp 63-79

8. De Vos P, Straaten JF van, Nieuwenhuizen AG et al. (1999) Why do microencapsulated islet grafts fail in the absence of fibrotic overgrowth? Diabetes 48:1381-1388

9. Klöck G, Frank H, Houben R et al. (1994) Production of purified alginates suitable for use in immunoisolated transplantation. Appl Microbiol Biotechnol 40:638-643

10. De Vos P, De Haan B, Van Schilfgaarde R (1997) Effect of the alginate composition on the biocompatibility of alginate-polylysine microcapsules. Biomaterials 18:273-278 
11. De Vos P, De Haan BJ, Wolters GHJ, Van Schilfgaarde R (1996) Factors influencing the adequacy of microencapsulation of rat pancreatic islets. Transplantation 62:888-893

12. De Vos P, De Haan BJ, Pater J, Van Schilfgaarde R (1996) Association between capsule diameter, adequacy of encapsulation, and survival of microencapsulated rat islet allografts. Transplantation 62:893-899

13. De Vos P, De Haan BJ, Wolters GHJ, Strubbe JH, Van Schilfgaarde R (1997) Improved biocompatibility but limited graft survival after purification of alginate for microencapsulation of pancreatic islets. Diabetologia 40:262270

14. Soon-Shiong P, Feldman E, Nelson R et al. (1992) Succesful reversal of spontaneous diabetes in dogs by intraperitoneal microencapsulated islets. Transplantation 54(5):769-774

15. Sun YL, Ma XJ, Zhou DB, Vacek I, Sun AM (1996) Normalization of diabetes in spontaneously diabetic cynomologus monkeys by xenografts of microencapsulated porcine islets without immunosuppression. J Clin Invest 98:14171422

16. Fritschy WM, De Vos P, Groen H et al. (1994) The capsular overgrowth on microencapsulated pancreatic islet grafts in streptozotocin and autoimmune diabetic rats. Transpl Int 7:264-271

17. Wolters GH, Suylichem PT van, Van Deijnen JH, Van Schilfgaarde R (1990) Factors influencing the isolation process of islets of Langerhans. Horm Metab Res [Suppl] 25:20-26

18. Suylichem PT van, Strubbe JH, Houwing H, Wolters GH, Van Schilfgaarde R (1994) Rat islet isograft function. Effect of graft volume and transplantation site. Transplantation 57:1010-1017

19. Suylichem PT van, Wolters GH, Van Schilfgaarde R (1990) The efficacy of density gradients for islet purification: a comparison of seven density gradients. Transpl Int 3:156-161

20. Wolters GHJ, Fritschy WM, Gerrits D, Van Schilfgaarde R (1992) A versatile alginate droplet generator applicable for microencapsulation of pancreatic islets. J Appl Biomaterials 3:281-286

21. Fritschy WM, Wolters GH, Van Schilfgaarde R (1991) Effect of alginate-polylysine-alginate microencapsulation on in vitro insulin release from rat pancreatic islets. Diabetes 40:37-43

22. Fritschy WM, Gerrits PO, Wolters GH, Pasma A, Van Schilfgaarde R (1995) Glycol methacrylate embedding of alginate-polylysine microencapsulated pancreatic islets. Biotech Histochem 70:188-193

23. Dijkstra CD, Dopp EA, Joling P, Kraal G (1985) The heterogeneity of mononuclear phagocytes in lymphoid organs: distinct macrophage subpopulations in the rat recognized by monoclonal antibodies ED1, ED2 and ED3. Immunology 54:589-599

24. Deenen GJ, Hunt SV, Opstelten D (1987) A stathmokinetic study of B lymphocytopoiesis in rat bone marrow: proliferation of cells containing cytoplasmic mu-chains, terminal deoxynucleotidyl transferase and carrying HIS24 antigen. J Immunol 139:702-710
25. Goor H van, Fidler V, Wening JJ, Grond J (1991) Determinants of focal and segmental glomerulosclerosis in the rat after renal ablation. Lab Invest 64:754-765

26. Huning T, Wallny HJ, Hartly J, Lawetsky A, Tiefenthaler G (1989) A monoclonal antibody to a constant region of the rat TCR that induces T-cell activation. J Exp Med 169:73-78

27. Reynolds CW, Sharrow SO, Ortaldo JR, Heberman RB (1981) Natural killer activity in the rat. II. Analysis of surface antigens on LGL by flow cytometry. J Immunol 127:2204-2205

28. Vos TA, Gouw AS, Klok PA et al. (1997) Differential effects of nitric oxide synthase inhibitors on endotoxin-induced liver damage in rats. Gastroenterology 113:1323-1333

29. Hamrick TS, Havell EA, Horton JR, Orndorff PE (2000) Host and bacterial factors involved in the innate ability of mouse macrophages to eliminate internalized unopsonizedEscherichia coli. Infect Immun 68:125-132

30. Helmke RJ, Boyd RL, German VF, Mangos JA (1987) From growth factor dependence to growth factor responsiveness: the genesis of an alveolar macrophage cell line. In Vitro Cell Dev Biol 23:567-574

31. Wang T, Lacík I, Brissová M et al. (1997) An encapsulation system for the immunoisolation of pancreatic islets. Nature Biotechnol 15:358-362

32. Strand BK, Ryan L, In 't Veld P et al. (2001) Poly-L-lysine induces fibrosis on alginate microcapsules via the induction of cytokines. Cell Transplant 10:263-277

33. Chen MC, Proost P, Gysemans C, Mathieu C, Eizirik DL (2001) Monocyte chemoattractant protein-1 is expressed in pancreatic islets from prediabetic NOD mice and in interleukin-1 beta-exposed human and rat islet cells. Diabetologia 44:325-332

34. Xiong M, Elson G, Legarda D, Leibovich SJ (1998) Production of vascular endothelial growth factor by murine macrophages: regulation by hypoxia, lactate, and the inducible nitric oxide synthase pathway. Am J Pathol 153:587-598

35. Wilander E, Boquist L, Rerup C, Falkmer S (1970) Functional and morphological studies of islet beta-cell regeneration in endogenous and exogenous diabetes. Acta Pathol Microbiol Scand [A] 78:496

36. Hsu BR, Chang FH, Juang JH, Huang YY, Fu SH (1999) The rescue effect of 15-deoxyspergualin on intraperitoneal microencapsulated xenoislets. Cell Transplant 8:307-315

37. Chicheportiche D, Reach G (1988) In vitro kinetics of insulin release by microencapsulated rat islets: effect of the size of the microcapsules. Diabetologia 31:54-57

38. Tziampazis E, Sambanis A (1996) Engineering a normally responsive bioartificial pancreas based on glucose-hypersensitive cells. Comput Biol Med 26:231-240

39. Tziampazis E, Sambanis A (1995) Tissue engineering of a bioartificial pancreas: modeling the cell environment and device function. Biotechnol Prog 11:115-126

40. Pavlovic D, Andersen NA, Mandrup-Poulsen T, Eizirik DL (2000) Activation of extracellular signal-regulated kinase (ERK)1/2 contributes to cytokine-induced apoptosis in purified rat pancreatic beta-cells. Eur Cytokine Netw $11: 267-274$ 